\title{
Isolated gonadotropin deficiency with primary empty sella: Causal or casual association?*
}

\author{
Shashank Shekhar ${ }^{1 \#}$, Chanderdeep Sharma ${ }^{1}$, Mukesh Surya ${ }^{2}$, Neeti Aggarwal ${ }^{3}$ \\ ${ }^{1}$ Department of Obstetrics \& Gynecology, Dr RPGMC Tanda, Kangra, India \\ ${ }^{2}$ Department of Radiodiagnosis, Dr RPGMC Tanda, Kangra, India \\ ${ }^{3}$ Indira Gandhi Medical College (IGMC), Shimla, India \\ Email: " longshanks28@gmail.com
}

Received 15 June 2012; revised 21 July 2012; accepted 1 August 2012

\begin{abstract}
Empty sella is a radiological-anatomical entity characterised by a sella turcica filled partially or completely with cerebrospinal fluid, leading to varying degrees of pituitary flattening. Literature reports a $5 \%-20 \%$ incidence of empty sella in unselected autopsies leading many to argue that it is an extreme normal variant. Endocrine dysfunction varies from $8 \%-25 \%$ with primary empty sella and growth hormone deficiency is most common. However isolated gonadotropin deficiency with normal sized primary empty sella is rare if any. We are reporting a case of isolated gonadotropin deficiency with primary empty sella unable to confirm or refute the association as causal or casual.
\end{abstract}

Keywords: Empty Sella; Empty Sella Syndrome; Gonadotropin Deficiency

\section{INTRODUCTION}

Empty sella is a radiological-anatomical entity characterised by a sella turcica filled partially or completely with cerebrospinal fluid (CSF), leading to varying degrees of pituitary flattening [1]. Thus, sella turcica is not empty as the name suggests, rather filled by a flattened pituitary, arachnoid and CSF. Based on eitio-pathogenesis empty sella is classified as primary and secondary. Empty sella is secondary when seen after surgery, irradiation or medical treatment of pituitary tumors or due to infective, autoimmune or traumatic causes. In the absence of such features empty sella is termed primary $[1,2]$. Two other terms used loosely and interchangeably in literature when describing empty sella are empty sella syndrome (ESS) and enlarged empty sella (EES). Association of empty sella with varying combination of clini-

\footnotetext{
${ }^{*}$ Conflict of interest: The authors report no conflicts of interest.
}

"Corresponding author. cal feature like hypopituitarism, headaches, visual disturbances and non traumatic CSF rhinorrhea constitutes the so called empty sella syndrome, whereas enlarged empty sella refers to empty sella with sellar volume exceeding $1.2 \mathrm{~cm}^{2}$ and may be associated with other features like marked asymmetry, ballooning, osseous erosions or defects $[3,4]$. Therefore, an empty sella may be of normal size and volume or enlarged when it is likely to be associated with symptoms. We describe here a normal sized empty sella associated with isolated gonadotropin deficiency presenting as primary amenorrhea and infertility in a young female. Whether the association was casual or causal could not be explained by radiological and endocrinological findings alone.

\section{CASE REPORT}

A 23 year old woman of Nepalese origin was evaluated for primary amenorrhea. Her developmental history was normal. History was negative for eating disorder, neurological episode, chronic illness or head trauma in the past. Subject denied history of chemo-irradiation or brain surgery in the past. History of chronic drug use or substance abuse was negative. Consanguinity in the family was denied. There was no other family member affected by absent puberty or infertility. Patient was $146 \mathrm{~cm}$ tall and weighed $55 \mathrm{~kg}$. Her breasts were Tanner's Stage IV and pubic hair was Tanner's II. There was no breast activity. Speculum examination revealed a healthy looking vagina and cervix. A small uterus was palpable on bimanual examination. Tests of olfaction were normal. Pelvic sonogram revealed a small uterus with normally visualized ovaries. A thorough ophthalmic, neurological and endocrine evaluation was done. Serum FSH (6 IU/L) and LH (4.6 IU/L) were normal. Fasting serum levels of GH (3.2 ng/ml), TSH $(2.3 \mu \mathrm{IU} / \mathrm{ml})$, Prolactin $(6 \mathrm{ng} / \mathrm{ml})$, ACTH $(26 \mathrm{pg} / \mathrm{ml})$ and IGF-1 $(46 \mathrm{ng} / \mathrm{ml})$ were normal. Serum estradiol was $36 \mathrm{pg} / \mathrm{ml}$. Serum cortisol $(9.4 \mu \mathrm{g} / \mathrm{dl}$ ) and 24 hour free urinary cortisol was in normal range. Serum screening for anti TPO antibodies was negative. 
Magnetic resonance imaging (MRI) revealed a flattened anterior pituitary against the floor of the sella turcica and CSF filling more than $50 \%$ of the sellar volume. Stalk of the pituitary was central. Posterior pituitary was normal (Figures 1-3). Olfactory bulbs and tracts were normally

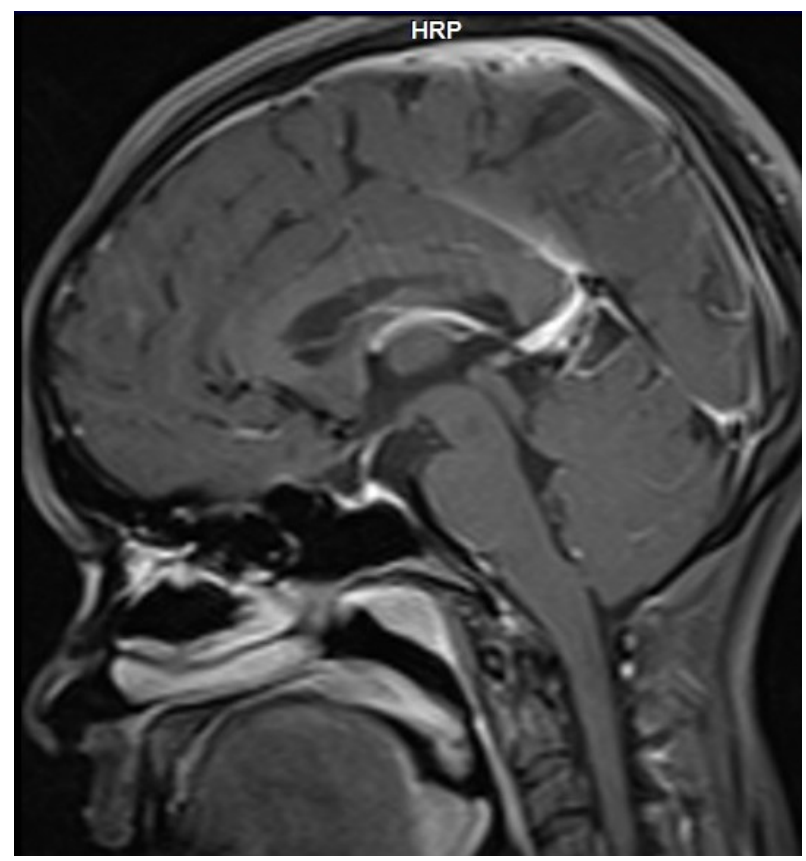

Figure 1. Post gadolinium T1 weighted magnetic resonance image of pituitary in sagittal section showing cerebrospinal filled sella turcica and a flattened anterior pituitary.

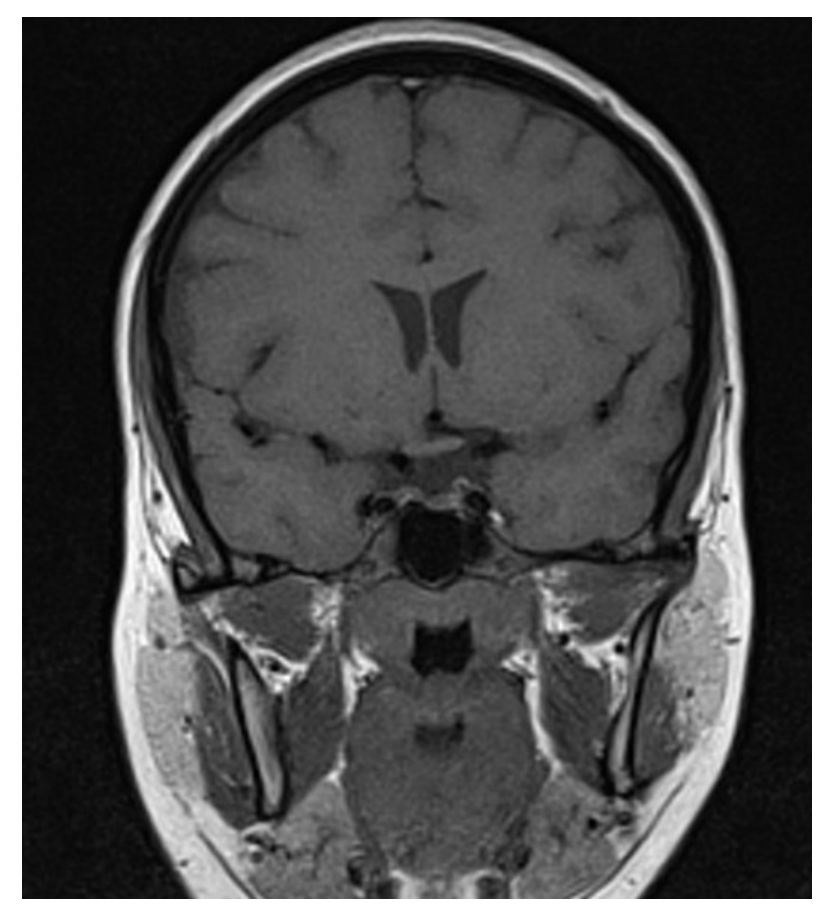

Figure 2. T1 weighted coronal magnetic resonance image of pituitary showing sella turcica filled with suprasellar subarachnoid space and flattened anterior pituitary.

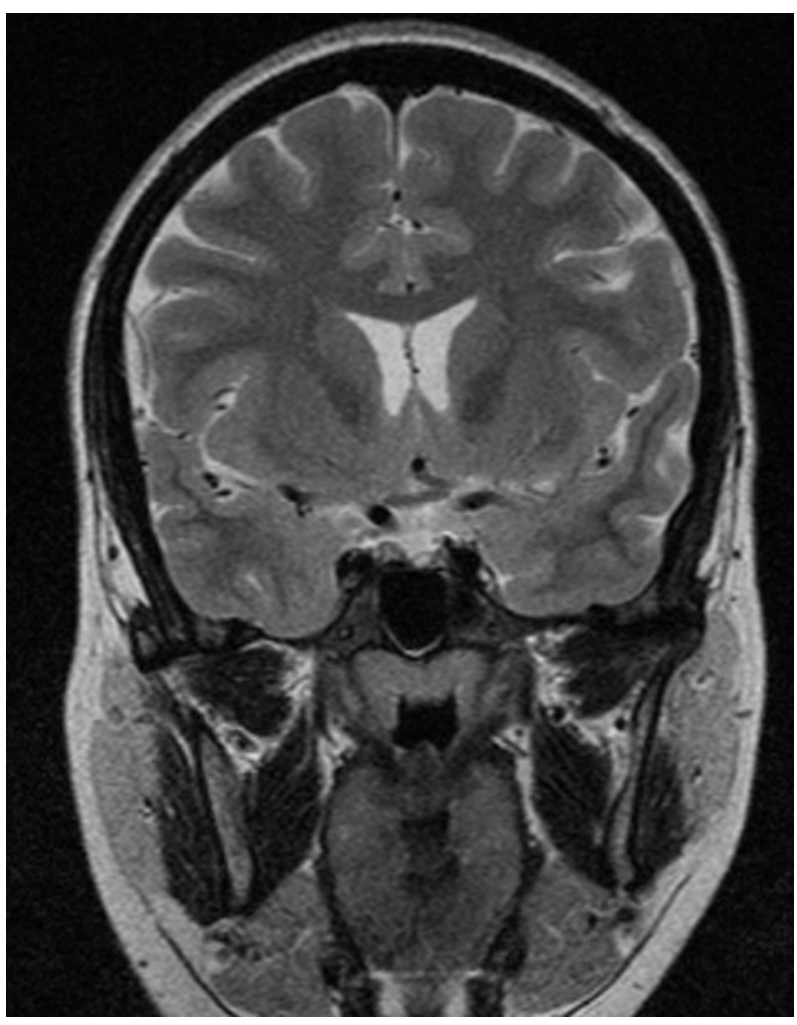

Figure 3. T2 weighted coronal magnetic resonance image of pituitary showing empty sella turcica.

visualized. Ophthalmic examination revealed no abnormality.

\section{DISCUSSION}

A number of hypotheses, explaining occurrence of primary empty sella (PES) include; necrosis/infarction of a pituitary adenoma, pituitary apoplexy, rupture of intrasellar cyst and volumetric changes associated with repeated pregnancies and aging. One of the most accepted explanation is congenitally deficient sellar diaphragm being acted upon by a sustained or intermittently increased intracranial pressure thus promoting herniation of arachnoid membrane into sella turcica [5]. Endocrine abnormalities are infrequent in subjects with PES. The spectrum of endocrine dysfunctions varies from isolated/ partial or global pituitary hyposecretion to hypersecretion. Whereas many authors [6] report up to $25 \%$ incidence of hypopituitarism with PES, the largest clinical case series of PES till date has reported 8\% incidence of global or partial pituitary hypofunciton [7]. In the same largest clinical case series none had isolated gonadotropin deficiency. The most common endocrine dysfunction reported with PES is GH deficiency either isolated or in combination with other anterior pituitary hormone hyposecretion. Basal serum GH levels are not clearly useful as most recent studies have demonstrated a 
decreased pituitary GH reserves in 35\% - 60\% of adult PES subjects [7-9]. IGF-1 levels and dynamic tests for GH reserves are more informative and useful. Although we could not perform dynamic tests of GH reserves nonetheless IGF-1 value was normal. Hyper-prolactinemia occurs in $10 \%$ of cases and theoretical explanation is stalk dysfunction possibly due to remodelling of the hypothalamo-pituitary region and altered CSF dynamics. The reported cases of empty sella with isolated gonadotropin deficiency in literature are rare. It has been postulated that different processes (vascular, autoimmune or genetic) which culminate in common final result of empty sella cause pituitary hypoplasia, hence pituitary insufficiency. In addition increased intrasellar pressure causing unabated pituitary compression and stalk distortion might lead to functional insufficiency of the pituitary cells and hypothalamic signal interference [10]. Hence, theoretically it should lead to at least partial pituitary insufficiency rather than an isolated hormonal deficiency. However, isolated GH deficiency is explained by the fact that somatotrophs constitute more than $50 \%$ of normal pituitary gland volume; hence their function is likely to be disturbed first in the case of pituitary damage. It is further hypothesized that the anatomical distribution of GH-secreting cells makes these cells more vulnerable and that the neuro-regulatory mechanisms of GH secretion may be particularly sensitive to increased intrasellar pressure [10]. However, isolated deficiency of gonadotropins with other pituitary hormones being normal is a rare presentation in this patient, which could not be explained well by the radiological findings alone. Despite a thorough ophthalmic, neurological and endocrine evaluation we could not establish a causal relationship between PES and isolated gonadotropin deficiency in this patient. As aptly quoted by someone "absence of proof is not proof of absence", hence, subjects with PES should be offered thorough clinical, hormonal and radiological evaluation.

\section{REFERENCES}

[1] McLachlan, M.S.F., William, E.D. and Doyle, F.H. (1968)
Applied anatomy of the pituitary gland and fossa: A radiological and histopathological study based on 50 necropsies. British Journal of Radiology, 41, 782-788. doi:10.1259/0007-1285-41-490-782

[2] Jordan, R.M., Kendall, J.W. and Kerber, C.W. (1977) The primary empty sella syndrome: Analysis of the clinical characteristic, radiographic features, pituitary function and cerebral fluid adeno-hypophysial concentrations. American Journal of Medicine, 62, 569-580. doi:10.1016/0002-9343(77)90420-X

[3] DiChiro, G. and Nelson, K.B. (1952) The volume of the sella turcica. American Journal of Roentgenology, 87, 989-1008.

[4] Bjerre, P. (1990) The empty sella. A reappraisal of etiology and pathogenesis. Acta Neurologica Scandinavica, 130, 1-25.

[5] Bragagni, G., Bianconcini, G., Mazzali, F., Baldini, A., Brogna, R., Iori, I., et al. (1995) 43 cases of primary empty sella syndrome: A case series. Annali Italiani di Medicina Interna, 10, 138-142.

[6] Arai, H. (2006) Empty sella syndrome. Nippon Rinsho, 212-216.

[7] De Marinis, L., Bonadonna, S., Bianchi, A., Maira, G. and Giustina, A. (2005) Extensive Clinical experience: Primary empty sella. The Journal of Clinical Endocrinology \& Metabolism, 90, 5471-5477. doi:10.1210/jc.2005-0288

[8] Gasperi, M., Aimaretti, G., Cecconi, E., Colao, A., Di Somma, C., Cannavo, S., et al. (2000) Impairment of GH secretion in adults with primary empty sella. Journal of Endocrinological Investigation, 25, 329-333.

[9] Cannavo, S., Curto, L., Venturino, M., Squardrito, S., Al Moto, B., Narbone, M.C., et al. (2002) Abnormalities of hypothalamic-pituitary-thyroid axis in patients with primary empty sella. Journal of Endocrinological Investigation, 25, 236-239.

[10] Del Monte, P., Foppiani, L., Cafferata, C., Marugo, A. and Bernasconi, D. (2006) Primary empty sella in adults: Endocrine findings. Endocrine Journal, 53, 803-809. doi:10.1507/endocrj.K06-024 